\title{
Parafluidity in Helium Near the $\lambda$-Transition and the Generalized Time-Dependent Landau Theory
}

\author{
D. Stauffer \\ Physik-Department der Technischen Hochschule \\ 8 München 2, Arcisstr. 2I, W. Germany \\ and Victor K. Wong* \\ Department of Physics, The University of Michigan \\ Ann Arbor, Michigan
}

(Received January 12, 1970)

A phenomenological theory of parafluidity, i.e., an enhancement of fluidity due to order-parameter fluctuations, is presented for helium near the $\lambda$ transition. The generalized time-dependent Landau theory of second-order phase transitions is reviewed in general and is applied to the superfluid transition in helium as a particular example. In helium, it is found that parafluidity is manifested in the divergences of the mass diffusivity $\mathrm{D}_{\mathrm{m}} \propto \varepsilon^{-\frac{1}{3}}$, the thermal conductivity $\kappa \propto \varepsilon^{-\frac{7}{3}}$, the first-sound amplitude attenuation $\alpha \propto|\varepsilon|^{-1}$, and the second-sound damping $\mathrm{D}_{2} \propto(-\varepsilon)^{-\frac{1}{3}}$, which are all consistent with the dynamic scaling hypothesis. Here a characteristic relaxation time $\tau_{0} \propto|\varepsilon|^{-1}$ is used, where $\varepsilon=\left(\mathrm{T}-\mathrm{T}_{\mathrm{c}}\right) / \mathrm{T}_{\mathrm{c}}$ and $\mathrm{T}_{\mathrm{c}}$ is the transition temperature. Although there are not enough experimental data to confirm our formulas, the present approach is seen to agree in order of magnitude with available experiments. Finally, the sound absorption above a ferromagnetic transition is calculated by adding a diffusion term to the generalized time-dependent Landau equation. The result thus obtained agrees in order of magnitude with experiments in nickel.

\section{INTRODUCTION}

The classical Landau theory ${ }^{1}$ of second-order phase transitions, which introduces the universal concept of the order parameter, is extremely useful for a qualitative understanding of the phase transition. In many systems, however, the classical Landau theory is quantitatively incorrect, since the classical critical exponents ${ }^{2,3}$ are not observed in experiments. On the other hand, the scaling laws of Widom, ${ }^{5}$ Kadanoff, ${ }^{6}$ and others ${ }^{2}$ are well satisfied by systems with nonclassical critical exponents. We can reconcile the classical Landau theory and the scalinglaw approach by noting that each has its own range of validity. ${ }^{2}$ In general the

\footnotetext{
*Supported in part by the National Science Foundation and the Horace H. Rackham School of Graduate Studies.
} 
classical range, where the classical Landau theory is valid, is close but not too close to the transition, whereas the critical range, where the scaling laws apply, is in the immediate vicinity of the transition. Despite its failings in the critical range, the Landau theory has an advantage over the scaling-law approach: the Landau theory can predict not only the temperature dependence of the critical behavior, i.e., critical exponents, but also the magnitude. ${ }^{7}$ Amit and Luban, ${ }^{8}$ Wong, ${ }^{9}$ and others $^{10,11}$ have been able to generalize the Landau theory so that the predictions of the generalized Landau theory agree with that of the scaling laws in the critical range. Furthermore, Stauffer ${ }^{11}$ has shown that the predictions of the generalized Landau theory agree in magnitude within $50 \%$ with experimental results of the phase transitions in ferromagnets, classical gases, quantum gases, binary mixtures, and superfluid helium.

The above considerations are restricted to static phenomena near the transition. An extension of the scaling-law approach to critical dynamic phenomena has been formulated by Ferrell et al., ${ }^{12}$ and Halperin and Hohenberg. ${ }^{13}$ Likewise, our purpose is to extend the generalized Landau theory to critical dynamic phenomena, using the $\lambda$ transition in helium as a prototype. In this manner, we hope to understand quantitatively, for example, the observed anomalous increase in the thermal conductivity of helium as the $\lambda$ transition is approached from above.

In Section 2 we formulate the generalized time-dependent Landau theory. The theory is applied in Section 3 to the calculation of the thermal conductivity and mass diffusivity in helium I near the $\lambda$ transition. The resultant divergence in these dynamic properties is a manifestation above the superfluid transition of parafluidity, or greatly enhanced fluidity due to order-parameter fluctuations. In Section 4 we calculate the contribution of order-parameter fluctuations to firstsound absorption in helium I, extend the calculation to helium II, and make contact with second-sound damping. As another application of the theory, we consider in Section 5 the sound absorption above the ferromagnetic transition and compare the prediction with experiments in nickel. A brief conclusion follows (Section 6).

\section{GENERALIZED TIME-DEPENDENT LANDAU THEORY}

The generalized Landau expansion ${ }^{8-11}$ for the free-energy functional $F\{\psi\}$ is

$$
\begin{gathered}
F\{\psi\}=\int d \mathbf{r}\left[f_{0}+A_{0}|\nabla \psi|^{2}+A|\psi|^{2}+B|\psi|^{4}+C|\psi|^{6}+\cdots\right] \\
\psi /|\varepsilon|^{\beta} \lesssim \text { constant, } \quad|\mathbf{k}| \xi \lesssim 1
\end{gathered}
$$

where $\psi$ is the order parameter; $f_{0}$ is the free-energy density of the unordered phase; $\varepsilon=\left(T-T_{c}\right) / T_{c} ; T_{c}$ is the critical temperature; $A_{0} \propto|\varepsilon|^{-\eta v}, A \propto|\varepsilon|^{\gamma}$, $B \propto|\varepsilon|^{\gamma-2 \beta}, \ldots ; \xi$ is the amplitude correlation length given by $\left(A_{0} / A\right)^{\frac{1}{2}}$ above $T_{c}, \mathbf{k}$ is a wave vector, and all the critical exponents are defined as in Reference 2. To be concrete, we consider $\psi$ to be complex as in helium. The order parameter is 
determined by the stationary condition

$$
\delta F\{\psi\} / \delta \psi^{*}=0
$$

which gives the relation

$$
L\{\psi\} \psi \equiv\left[-A_{0} \nabla^{2}+A+2 B|\psi|^{2}+3 C|\psi|^{4}+\cdots\right] \psi=0
$$

if the surface integrals

$$
\int \delta \psi^{*} A_{0} \nabla \psi \cdot d \sigma+\text { complex conjugate }
$$

are discarded. The surface integrals $(2 \mathrm{c})$ vanish if, at the surface,

$$
(\nabla \psi)_{n}=0
$$

which is satisfied for an isolated superconductor. ${ }^{7}$ For helium, the correct boundary condition at a surface is probably closer to $\psi=0$. In the present work, we ignore the surface integrals.

In the critical range, the calculation of static phenomena ${ }^{11}$ based on (1) and (2) consists essentially of rewriting the classical results with general critical exponents. Similarly to calculate dynamic properties, we rewrite the classical timedependent Landau theory with general critical exponents.

The classical time-dependent Landau theory ${ }^{14,15}$ assumes that the order parameter relaxes to its equilibrium value according to

$$
\partial \psi / \partial t=-R_{c l} \cdot L_{c l}\{\psi\} \psi
$$

where the classical relaxation coefficient $R_{c l}$ is a constant and $L_{c l}\{\psi\}$ is given by (2b) with the classical exponents and without the terms $C|\psi|^{4}+\cdots$. We now rewrite (3) with general critical exponents

$$
\partial \psi / \partial t=-R \cdot L\{\psi\} \psi
$$

where the temperature-dependent relaxation coefficient $R$ is to be taken from experiments, and $L\{\psi\} \psi$ is given by (2b). In general, the temperature dependence of $R$ cannot be related to the critical exponents because of the existence of different transport modes with differing frequencies, strengths, and decay rates in the various phase transitions. However, for a particular system the critical exponent of $R$ can be thus related $;^{16}$ e.g., in the two-dimensional Ising model, Suzuki et al. ${ }^{17}$ have indicated that $R \propto \varepsilon^{\frac{1}{4}}$. Equation (4) has been proposed previously in calculations on the first-sound absorption in helium by Prokovskii and Khalatnikov ${ }^{18}$ and by Wong $^{19}$; and the agreement of the predictions of (4) with the dynamic scaling hypothesis ${ }^{12,13}$ was pointed out by DiCastro et al. ${ }^{20} \mathrm{We}$ call Eq. (4) the generalized time-dependent Landau equation, and the ensuing theory the generalized timedependent Landau theory.

To supplement Eq. (4) we write down the expression for the order-parameter current density below $T_{c}$ in the limit of zero field:

$$
\mathbf{j}^{\psi}(\mathbf{r}, t)=\left(A_{0} / \hbar i\right)\left(\psi^{*} \nabla \psi-\psi \nabla \psi^{*}\right)
$$

It is easy to verify that $\mathrm{j}^{\psi}=n_{s} \mathbf{v}_{s}$ with the superfluid number density ${ }^{21}$ $n_{s}=\left(2 m A_{0} / h^{2}\right)|\psi|^{2}$, and the superfluid velocity $\mathbf{v}_{s}=(\hbar / m) \nabla($ phase of $\psi)$, where $m$ is some mass (e.g., for helium $m$ is equal to the mass of the helium atom). It is 
customary to define a superfluid density $\rho_{s}=m n_{s}$ and the current density $\mathbf{j}=m \mathbf{j}^{\psi}$. Then it is straightforward to show that $\mathbf{j}=\rho_{s} \mathbf{v}_{s}$; and for a system with uniform $|\psi|, A_{0}|\nabla \psi|^{2}=\frac{1}{2} \rho_{s} v_{s}^{2}$. Above $T_{c}$, Eq. (5) is still applicable, since the meansquare value $\left\langle|\psi|^{2}\right\rangle$ [cf. Eq. (6) below] is nonzero as a result of fluctuations, and the quantity $\rho_{p}=\left(2 m^{2} A_{0} / \hbar^{2}\right)\left\langle|\psi|^{2}\right\rangle$ can be interpreted as the parafluid density inside fluctuations of volume $\xi^{3}$, which has a finite lifetime in accordance to (4). Since parafluidity will be discussed in detail in Section 3, it is only necessary at this point to recognize that (5) can be successfully applied on both sides of the phase transition.

In the classical range, the use of the classical equation (3) or its equivalence has led to the successful predictions of anomalous fluctuation effects in dynamic properties. For example, above $T_{c}$ the electric conductivity and the diamagnetic susceptibility in a bulk superconductor ${ }^{22-26}$ and the thermal conductivity in a van der Waals gas ${ }^{27}$ have been shown to diverge as $\varepsilon^{-\frac{1}{2}}$. The phenomenological approach of $\mathbf{H}$. Schmidt ${ }^{25}$ for the electrical conductivity of a superconductor is most useful because it is in complete correspondence with the microscopic theory of Aslamazov and Larkin. ${ }^{22}$ An essential step in the calculation of H. Schmidt, ${ }^{25}$ which is not used in two other phenomenological calculations, ${ }^{23,24}$ is a Kubo-type formula that relates the electrical conductivity to the current fluctuations. By means of (5) with $A_{0}=\hbar^{2} / 2 m$, the current fluctuations are expressed in terms of the orderparameter fluctuations, which are in turn calculated by the time-dependent Ginzburg-Landau equation, i.e., Eq. (3). These considerations for a superconductor in the classical range have been emphasized recently by Ferrell. ${ }^{26}$

In the critical range, we calculate the dynamic properties in the generalized time-dependent Landau theory by following the procedure used by $\mathrm{H}$. Schmidt, ${ }^{25}$ but with (4) replacing (3). In the examples treated here, the difference between the present procedure and the plausibility argument of DiCastro et al ${ }^{20}$ (in the classical range, between $H$. Schmidt ${ }^{25}$ and others ${ }^{23,24}$ ) is not an essential one; however the difference is significant for a superconductor in a nonzero magnetic field. ${ }^{28,29}$

\section{PARAFLUIDITY IN HELIUM ABOVE THE SUPERFLUID TRANSITION}

As far as we know, the word paraconductivity was first used by Ferrell ${ }^{26}$ to describe the diverging electric conductivity above the superconducting transition, in loose analogy with the diverging paramagnetic susceptibility of a ferromagnet above the Curie point. Similarly, in a superfluid transition we use parafluidity to mean the enhanced fluidity due to order-parameter fluctuations with finite lifetimes and finite ranges, and parafluidity is manifested above $T_{c}$ in diverging thermal conductivity and mass diffusivity. The major difference between paraconductivity and parafluidity, as far as calculations are concerned, is that the former occurs in the classical range, whereas the latter occurs in the critical range. This difference necessitates the use of the generalized time-dependent Landau equation (4) to describe parafluidity. 


\subsection{Plausibility Arguments}

Parafluidity can be made plausible ${ }^{20}$ by analogy with the arguments used for paraconductivity. ${ }^{23,24,26}$ In helium I $\left(T \gtrsim T_{c}\right)$, spontaneous order-parameter fluctuations produce islands of superfluidity $(\psi \neq 0)$ which cannot persist but must relax to the equilibrium value, $\langle\psi\rangle=0$, in accordance with (4). The typical fluctuation has a finite range of the order of $\xi=\left(2 A_{0} \chi_{0}\right)^{\frac{1}{2}}$ and a finite lifetime of the order of $\tau_{0}=2 \chi_{0} R^{-1}$, after which a new set of fluctuations will replace the preceding ones. Here $\chi_{0}$ is the static susceptibility given by $\chi_{0}^{-1}=\partial^{2} F / \partial \psi^{2}=$ $2 A+\cdots$. The finite lifetime $\tau_{0}$ leads to the following estimate of anomalous transport. The mean-square fluctuation,

$$
\left\langle|\psi|^{2}\right\rangle=(\Delta Z)^{-1} \int d \psi|\psi|^{2} \exp \left(-\Delta F\{\psi\} / k_{B} T\right)
$$

where

$$
\left\{\begin{aligned}
\Delta Z & =\int d \psi \exp \left(-\Delta F\{\psi\} / k_{B} T\right) \\
\Delta F\{\psi\} & =F\{\psi\}-F\{\langle\psi\rangle\}
\end{aligned}\right.
$$

is given by the equipartition theorem as $\left\langle|\psi|^{2}\right\rangle \propto k_{B} T_{c} \chi_{0} \xi^{-3} \propto\left(A_{0} \xi\right)^{-1}$, where $k_{B}$ is the Boltzmann constant; and the mean density of the parafluid can be taken to be $\rho_{p}=\left(2 m^{2} / h^{2}\right) A_{0}\left\langle|\psi|^{2}\right\rangle \propto \xi^{-1}$. In helium II $\left(T \lesssim T_{c}\right)$, the thermal conductivity $\kappa$ is infinite and the mass flow through capillaries is frictionless. As a result of fluctuations, $\kappa$ and the mass diffusivity $D_{m}$ experiences in helium $I$ an anomalous mode of transport, whose contribution is proportional to the mean parafluid density times its mean lifetime, i.e., $\rho_{p} \tau_{0} \propto \tau_{0} \xi^{-1}$. Taking $\tau_{0} \propto\left|c_{2}^{-1} \xi\right| \propto$ $\varepsilon^{-1}$ and $\xi \propto\left|\rho_{s}^{-1}\right| \propto \varepsilon^{-\frac{3}{3}}$, where $c_{2}$ is the second-sound speed and $\rho_{s}$ the superfluid density, we find the parafluid contribution to $\kappa$ and $D_{m}$, which we distinguish by primes, to be

$$
\kappa^{\prime} \propto D_{m}^{\prime} \propto \tau_{0} \xi^{-1} \propto \varepsilon^{-\frac{1}{3}}
$$

Note that the temperature dependence of $\kappa^{\prime}$ agrees qualitatively with experiments. ${ }^{30,31}$ Similarly, the finite range $\xi$ of the order produced by the fluctuations results in an increase of the free energy, ${ }^{32}$ i.e.,

$$
F^{\prime}=-k_{B} T \ln \Delta Z \text {, }
$$

which can be reduced with the aid of (7) to $F^{\prime} \propto \xi^{-3} \ln \xi$. Taking $\xi \propto \varepsilon^{-\frac{3}{3}}$, we find the parafluid contribution to the specific heat $C_{p}$ to be

$$
C_{p} \propto \ln \left|\varepsilon^{-1}\right|
$$

which is in qualitative agreement with experiments. ${ }^{33,34}$ Although the restriction to wavelengths around $\xi$ in the reduction of $(9)$ to (10) is open to question, the authors feel that a previous attempt ${ }^{35}$ to incorporate the logarithmic singularity in $C_{p}$ is not correct, and tend towards the treatment based on (9). 


\subsection{Thermal Conductivity and Mass Diffusivity}

To calculate $\kappa$ and $D_{m}$ quantitatively, we begin with the Kubo-type formulae $\mathrm{e}^{36,37}$ which relate $\kappa$ and $D_{m}$ to the correlations of the heat current density $\mathrm{j}^{q}$ and the mass current density $\boldsymbol{j}^{\rho}$, respectively:

$$
\begin{gathered}
\kappa=\frac{1}{2 k_{B} T_{c}^{2}} \lim _{\omega \rightarrow 0}\left[\lim _{k \rightarrow 0}\left\langle j_{x}^{q}(\mathbf{k}, \omega) j_{x}^{q}(-\mathbf{k},-\omega)\right\rangle\right] \\
D_{m} \frac{\partial \rho}{\partial \mu}=\frac{1}{2 k_{B} T_{c}} \lim _{\omega \rightarrow 0}\left[\lim _{k \rightarrow 0}\left\langle j_{x}^{\rho}(\mathbf{k}, \omega) j_{x}^{\rho}(-\mathbf{k},-\omega)\right\rangle\right]
\end{gathered}
$$

where $\rho$ is the total mass density and $\mu$ is the chemical potential. We consider helium I near $T_{c}$ to be described by a quasi-two-fluid model: a parafluid with a finite lifetime, and the rest of the fluid, the normal fluid. The mass current density is $\mathbf{j}^{\rho}=\mathbf{j}+\rho_{n} \mathbf{v}_{n}$, where $\mathbf{j}$ can be interpreted as the parafluid current and is given by the mass of the helium atom, $m$, times the order-parameter current density (5); $\rho_{n}$ and $v_{n}$ are the density and velocity, respectively, of the normal fluid. The heat current density is taken to be $\mathbf{j}^{q}=\rho T S \mathbf{v}_{n}=T S\left(\rho / \rho_{n}\right)\left(\mathbf{j}^{\rho}-\mathbf{j}\right)$, where $S$ is the entropy per unit mass of the total fluid. In this manner, the parafluid contribution to both $\kappa$ and $D_{m}$ near $T_{c}$ is given by

$$
\frac{\kappa^{\prime}}{T_{c} S^{2}}=D_{m}^{\prime} \frac{\partial \rho}{\partial \mu}=\frac{1}{2 k_{B} T_{c}} \lim _{\omega \rightarrow 0}\left[\lim _{\mathbf{k} \rightarrow 0} \frac{1}{3}\left\langle\mathbf{j}_{k} \cdot \mathbf{j}_{-k}\right\rangle\right]
$$

where $\mathbf{j}_{k}$ is $m$ times the Fourier transform of (5):

$$
\mathbf{j}_{k}=\mathbf{j}(\mathbf{k}, \omega)=-\frac{2 m A_{0}}{\hbar} \sum_{k^{\prime}} \mathbf{k}^{\prime} \psi_{k^{\prime}-\frac{1}{2} k}^{*} \psi_{k^{\prime}+\frac{1}{2} k}
$$

with

$$
\begin{aligned}
k^{\prime} & \Rightarrow\left(\mathbf{k}^{\prime}, \omega^{\prime}\right) \\
\sum_{k^{\prime}} & \Rightarrow \int \frac{d \mathbf{k}^{\prime}}{(2 \pi)^{3}} \int \frac{d \omega^{\prime}}{2 \pi}
\end{aligned}
$$

and

$$
\psi_{k}=\psi(\mathbf{k}, \omega)=\int d \mathbf{r} \int d t[\exp (-i(\mathbf{k} \cdot \mathbf{r}-\omega t))] \psi(\mathbf{r}, t)
$$

Since the parafluid contribution to $D_{m}(\partial \rho / \partial \mu)$ is the only contribution in the case where the normal fluid does not fluctuate appreciably, we expect the result of (13) for $D_{m}^{\prime}(\partial \rho / \partial \mu)$ to be applicable to helium in a porous medium where motion in the normal fluid is damped out by viscosity. For thermal conductivity, the parafluid contribution is the main contribution in situations where the density fluctuations reflected in $\left\langle\mathbf{j}^{\rho} \cdot \mathbf{j}^{\rho}\right\rangle$ and the entropy fluctuations ${ }^{38}$ are negligible. Density fluctuations are not important ${ }^{13}$ except perhaps in an unaccessible temperature range very close to $T_{c}$; however, entropy fluctuations are not negligible in the experimentally 
accessible range for bulk helium. It is not clear a priori whether all factors of the specific heat $C_{p}$ will be in $\kappa^{\prime}$ as calculated from (13), in comparison with that from extended dynamic scaling, ${ }^{13}$ since $C_{p}$ can be considered as arising from the normalization of the entropy fluctuations ${ }^{38}$ which we have neglected. As we shall see, the logarithmic dependence of $\kappa^{\prime}$ from (13) agrees with the theoretical predictions of Reference 12 . The spirit of (13) is analogous to calculations of the electrical conductivity of a superconductor ${ }^{22-26}$ above $T_{c}$, in which only orderparameter fluctuations are considered since the lattice damps out any motion of the normal fluid.

Now we calculate the contribution of order-parameter fluctuations to $\langle\mathbf{j} \cdot \mathbf{j}\rangle$, in analogy to the classical calculation by $\mathrm{H}$. Schmidt. ${ }^{25}$ Using (14), we have

$$
\left\langle\mathbf{j}_{k} \cdot \mathbf{j}_{-k}\right\rangle=\left(\frac{2 m A_{0}}{\hbar}\right)^{2} \sum_{k^{\prime} k^{\prime \prime}} \mathbf{k}^{\prime} \cdot \mathbf{k}^{\prime \prime}\left\langle\psi_{k^{\prime}-\frac{1}{2} k}^{*} \psi_{k^{\prime \prime}+\frac{1}{2} k} \psi_{k^{\prime \prime}+\frac{1}{2} k}^{*} \psi_{k^{\prime \prime}-\frac{1}{2} k}\right\rangle
$$

If the fluctuations with different $k$ are statistically independent, we can factorize

$$
\left\langle\psi_{k^{\prime}-\frac{1}{2} k}^{*} \psi_{k^{\prime}+\frac{1}{2} k} \psi_{k^{\prime \prime}+\frac{1}{2} k}^{*} \psi_{k^{\prime \prime}-\frac{1}{2} k}\right\rangle=\delta_{k^{\prime} k^{\prime \prime}}\left\langle\psi_{k^{\prime}-\frac{1}{2} k}^{*} \psi_{k^{\prime}-\frac{1}{2} k}\right\rangle\left\langle\psi_{k^{\prime}+\frac{1}{2} k}^{*} \psi_{k^{\prime}+\frac{1}{2} k}\right\rangle
$$

and obtain

$$
\left\langle\mathbf{j}_{k} \cdot \mathbf{j}_{-k}\right\rangle=\left(\frac{2 m A_{0}}{h}\right)^{2} \sum_{k^{\prime}}\left|\mathbf{k}^{\prime}\right|\left\langle\psi_{k^{\prime}-\frac{1}{2} k}^{*} \psi_{k-\frac{1}{2} k}\right\rangle\left\langle\psi_{k^{\prime}+\frac{1}{2} k}^{*} \psi_{k^{\prime}+\frac{1}{2} k}\right\rangle
$$

which can be represented by Fig. 1. If the anharmonic terms in (1) can be ignored, we can calculate the time-independent mean-square fluctuations from (6) and (7) and get

$$
\begin{aligned}
\left\langle\psi_{\mathbf{k}}^{*} \psi_{\mathbf{k}^{\prime}}\right\rangle & =\delta_{\mathbf{k} \mathbf{k}^{\prime}} 2 k_{B} T_{c} \chi_{k} \\
\chi_{\mathbf{k}}^{-1} & =\chi_{0}^{-1}\left(1+|\mathbf{k}|^{2} \xi^{2}\right)
\end{aligned}
$$

where the static susceptibility $\chi_{0}$ is given by $\chi_{0}^{-1}=2 A+\cdots$, and the amplitudecorrelation length $\xi=\left(2 A_{0} \chi_{0}\right)^{\frac{1}{2}}$. To obtain the fluctuation spectrum near $T_{c}$, it is sufficient to consider a linear approximation to the generalized time-dependent Landau equation (4), which after Fourier transforming can be written as

$$
\begin{aligned}
& \left(-i \omega+\Gamma_{k}\right) \psi_{k}=0 \\
& \Gamma_{k}=\Gamma_{0}\left(1+|\mathbf{k}|^{2} \xi^{2}\right)
\end{aligned}
$$

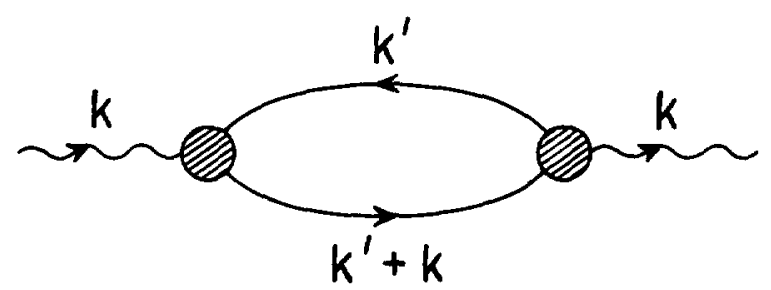

Fig. 1. Schematic representation of Eqs. (17) and (38). Decay of one transport mode $(\sim)$ into two order-parameter fluctuations $(\longrightarrow)$. 
where the relaxation frequency is given by $\Gamma_{0}=\tau_{0}^{-1}=R / 2 \chi_{0}$. In this manner, we obtain the fluctuation spectrum

$$
\begin{aligned}
\left\langle\psi_{k}^{*} \psi_{k^{\prime}}\right\rangle & =\delta_{k k^{\prime}} 2 k_{B} T_{c} \chi_{k} \\
\chi_{k} & =\chi_{k} 2 \Gamma_{\mathbf{k}} /\left(\Gamma_{\mathbf{k}}^{2}+\omega^{2}\right)
\end{aligned}
$$

such that

$$
\chi_{k}=\int \frac{d \omega}{2 \pi} \chi_{k}
$$

The conditions on (18) and (20) are restriction $|\mathbf{k}| \xi<1$ on (1), and the restriction $\omega \tau_{0}<1$ on (4). Substituting (20) into (17), we can perform the $\omega^{\prime}$ integration explicitly and obtain

$$
\left\langle\mathbf{j}_{k} \cdot \mathbf{j}_{k}\right\rangle=\left(\frac{2 m A_{0}}{\hbar}\right)^{2}\left(4 k_{B} T_{c} \Gamma_{0} \chi_{0}\right)^{2} \int \frac{d \mathbf{k}^{\prime}}{(2 \pi)^{3}} \mathbf{k}^{\prime} \cdot \mathbf{k}^{\prime} \frac{\Gamma_{+}+\Gamma_{-}}{2 \Gamma_{+} \Gamma_{-}} \frac{1}{\omega^{2}+\left(\Gamma_{+}+\Gamma_{-}\right)^{2}}
$$

where $\Gamma_{ \pm}=\Gamma_{\mathbf{k}^{\prime} \pm \frac{1}{2} \mathbf{k}}$. Equation (22) is the desired result for the current-correlation function.

Equation (22) can be substituted back onto (13) to give

$$
\frac{\kappa^{\prime}}{T_{c} S^{2}}=D_{m}^{\prime} \frac{\partial \rho}{\partial \mu}=\frac{8}{3}\left(\frac{m}{\hbar}\right)^{2} k_{B} T_{c}\left(A_{0} \Gamma_{0} \chi_{0}\right)^{2} \int \frac{d \mathbf{k}^{\prime}}{(2 \pi)^{3}} \frac{\left|\mathbf{k}^{\prime}\right|^{2}}{\Gamma_{\mathbf{k}}^{3}}
$$

Substituting (19) into (23) and performing the $\mathbf{k}^{\prime}$ integration, we obtain the final form :

$$
\frac{\kappa^{\prime}}{T_{c} S^{2}}=D_{m}^{\prime} \frac{\partial \rho}{\partial \mu}=\frac{1}{16 \pi}\left(\frac{m}{\hbar}\right)^{2} k_{B} T_{c} \frac{\tau_{0}}{\xi}=C_{0} \frac{\tau_{0}(\varepsilon)}{\xi(\varepsilon)}
$$

where $\tau_{0}(\varepsilon)$ and $\xi(\varepsilon)$ are to be evaluated for $T>T_{c}$ and $C_{0}=2.38 \times 10^{-10} \mathrm{~g} / \mathrm{cm}^{2}$.

Since the order parameter $\psi$ is not a constant of the motion above $T_{c}$, the frequency dependence of the order-parameter correlation function (20) cannot be determined from hydrodynamics. This has prevented a direct check of the restricted dynamic-scaling hypothesis. ${ }^{13}$ If we apply the asymptotic matching condition to the characteristic frequencies of the order-parameter correlation function above $T_{c}$ [Eq. (20)] and below $T_{c}$, assuming the dominance of the second-sound mode, we obtain $\Gamma\left(|\mathbf{k}|=\xi^{-1}\right) \sim c_{2} \xi^{-1} \sim \frac{1}{2} D_{2} \xi^{-2}$ or $\tau_{0}^{-1}(\varepsilon) \sim \frac{1}{2} c_{2} \xi^{-1} \sim \frac{1}{4} D_{2} \xi^{-2}$, where $c_{2} \propto\left(\rho_{s} / C_{p}\right)^{\frac{1}{2}} \propto \varepsilon^{\frac{1}{s}}$ is the second-sound speed, and $D_{2}$ is the second-sound damping. Note that the above relations were obtained solely from restricted dynamic scaling, unlike Reference 20 . Thus from (24) we have $\kappa^{\prime}(\varepsilon) \propto D_{m}^{\prime}(\varepsilon) \propto \varepsilon^{-\frac{1}{s}}$, which is in qualitative agreement with calculations based on mode-mode coupling, ${ }^{38,39}$ and for $\kappa^{\prime}(\varepsilon)$ with experiments in bulk helium. ${ }^{31}$ Determination of $\tau_{0}(\varepsilon)$ offers a direct check of the restricted dynamic-scaling hypothesis as applied to helium.

Quantitatively the assessment of (24) is less clear because of the scarcity of experimental results for $\tau_{0}(\varepsilon)$. In order to fit the measured thermal conductivity, 
we take $\xi(\varepsilon)=(1.6 \AA) \varepsilon^{-\frac{3}{3}}$ from Mamaladze $\mathrm{e}^{40}$ and

$$
\tau_{0}(\varepsilon) \approx 0.6 \times 10^{-10} \varepsilon^{-1} \mathrm{sec}
$$

Using (25) in (24), we get

$$
\kappa^{\prime}(\varepsilon) \approx 0.5 \times 10^{-4} \varepsilon^{-\dagger} \mathrm{W} / \mathrm{cm}^{\circ} \mathrm{K}
$$

which agrees to $30 \%$ with the bulk measurements of Ahlers, ${ }^{31}$ in the range $10^{-6}<\varepsilon<10^{-3}$. The estimate $(25)$ for $\tau_{0}(\varepsilon)$ is larger by about one order of magnitude than the estimate $\tau_{0}(\varepsilon) \approx 2 \xi / c_{2} \approx 2 \xi^{2} / D_{2}$. A better fit to $\kappa^{\prime}$ can be obtained by letting $\tau_{0}(\varepsilon) \propto \varepsilon^{-z}, z \lesssim 1$, or by logarithmic corrections to $\varepsilon^{-1}$. However, for order-of-magnitude comparisons it suffices to take $\tau_{0}(\varepsilon) \propto \varepsilon^{-1}$.

Gould and Wong ${ }^{41}$ have recently derived microscopically the parafluid contribution to the thermal conductivity and found $\kappa^{\prime}(\varepsilon) \propto \varepsilon^{-t}$. Thus the present work bears the same relation to Gould and Wong ${ }^{41}$ as the work of $\mathrm{H}$. Schmidt ${ }^{25}$ to that of Aslamazov and Larkin. ${ }^{22}$

The quantity $D_{m}^{\prime}(\partial \rho / \partial \mu)$ can be measured if a pressure gradient is applied to helium I in capillaries so narrow that no viscous flow is possible and only a homogeneous flow independent of the capillaries' radii occurs. The mass current density is then given by the parafluid contribution $\mathbf{j}=D_{m}^{\prime}(\partial \rho / \partial \mu) \nabla \mu$. The mean velocity induced by parafluidity is given by $\mathbf{v}_{p}=\mathbf{j} / \rho=\rho^{-1} D_{m}^{\prime}(\partial \rho / \partial \mu) \nabla \mu$. Using (25) in (24), we find that helium I under its own gravitational pressure $(\mu=g z)$ flows with parafluid speed

$$
v_{p}(\varepsilon)=0.6 \times 10^{-8} \varepsilon^{-\frac{1}{3}} \mathrm{~cm} / \mathrm{sec}
$$

which agrees with the plausibility arguments of Wilkins. ${ }^{42}$ No existing experimental test for (27) is known to us. Wilkins ${ }^{42}$ has attempted to extend the theory to a two- or one-dimensional geometry by taking into account only the reduction in phase space. A calculation including the effects of the boundary condition $\psi=0$ should be performed in order to evaluate the effects of parafluidity in restricted geometry.

The present calculation of the parafluid drift velocity, Eqs. (13)-(27), is necessary only if one desires to express the mass diffusivity in terms of $\tau_{0}$ and $\xi$. If instead the thermal conductivity $\kappa^{\prime}$ is used as a free parameter of the theory, then $\mathbf{v}_{p}$ can be directly expressed in terms of $\kappa^{\prime}$ by

$$
\mathbf{v}_{p}=\rho^{-1}\left(\kappa^{\prime} / T_{c} S^{2}\right) \nabla \mu
$$

The main assumption that is necessary to derive (28) is that the two-fluid formula for the heat current with zero flow in He II, $\mathbf{j}^{q}=\rho T S \mathbf{v}_{n} \approx-T S \mathbf{j}$, also holds for the finite-lifetime fluctuations in He I. A direct experimental test for the validity of $\mathrm{j}^{q}=\rho T S \mathbf{v}_{n}$ in He I would be to measure the drift velocity of impurities in $\mathrm{He} \mathrm{I}$ in a heat current. Assuming that the impurities move with the normal fluid, the drift speed close to the $\lambda$ transition is given by

$$
v_{n}=f^{q} / \rho T_{\mathfrak{c}} S
$$

which is equal to $2 \times 10^{-3} \mathrm{~cm} / \mathrm{sec}$ for $j^{q}=1 \mathrm{~mW} / \mathrm{cm}^{2}$. 
If we utilize the information from hydrodynamics and restricted dynamic scaling, we find that $\tau_{0}(\varepsilon) \propto \varepsilon^{-1}\left(C_{p}^{-}\right)^{\frac{1}{2}}$, where $C_{p}^{-}\left(C_{p}^{+}\right)$is the specific heat below (above) $T_{c}$. Then we find that $v_{p}(\varepsilon) \propto D_{m}^{\prime}(\varepsilon) \propto \kappa^{\prime}(\varepsilon) \propto \varepsilon^{-\frac{f}{5}}\left(C_{p}^{-}\right)^{\frac{1}{1}}$, which agrees with the theory of Reference 12, but is to be compared with $\kappa^{\prime}(\varepsilon) \propto \varepsilon^{-\frac{3}{5}} C_{p}^{+} /\left(C_{p}^{-}\right)^{\frac{1}{2}}$ from extended dynamic scaling. ${ }^{13}$

Below $T_{c}$, the effective thermal conductivity is extremely large and easily masks all parafluid effects. If we neglect the $\mathbf{k}=0, \omega=0$ component of the current, then the form of the current correlation function is the same as for above, i.e., as given by (13) and (24), but $\tau_{0}$ and $\xi$ are now the values below $T_{c}$. It has been suggested ${ }^{43}$ that the mass diffusion and perhaps also the spin diffusion of $\mathrm{He}^{3}$ impurities in $\mathrm{He}^{4}$ behave similarly to the thermal conductivity near $T_{c}$ and give an $|\varepsilon|^{-\frac{1}{3}}$ divergence ${ }^{13}$ on both sides of $T_{c}$. Such an experiment would yield information on the relaxation times above and below $T_{c}$ and verify the dynamic parafluid effects on both sides of $T_{c}$.

\subsection{Factorization of the Fluctuations}

We consider now in more detail the factorization (16) in the critical range, by analogy with the estimates in the classical range. ${ }^{26}$ After Fourier transforming, we can write the free energy (1) above $T_{c}$ as

$$
F\{\psi\}=F_{0}+\sum_{k}\left(A+A_{0}|\mathbf{k}|^{2}\right)\left|\psi_{k}\right|^{2}+\sum_{k^{\prime} k^{\prime \prime} k} B \psi_{k+k^{\prime \prime}}^{*}-k^{\prime} \psi_{k} \psi_{k^{\prime}}^{*} \psi_{k^{\prime \prime}}+\cdots
$$

If the anharmonic terms in (30) are negligible, then the correlation function $\left\langle\psi_{k}^{*} \psi_{k^{\prime}}\right\rangle$ is given by (18). We can estimate the correction due to the anharmonic terms by substituting (18) into $\sum_{k k^{\prime}} B\left\langle\psi_{k}^{*} \psi_{k}\right\rangle\left\langle\psi_{k}^{*} \psi_{k^{\prime}}\right\rangle$. As the minimum wavelength of interest is of the order $\xi$, we note that the anharmonic correction for fluctuations with wavevectors $|\mathbf{k}| \approx \xi^{-1}$ can be neglected if

$$
1 \gg \sum_{|\mathbf{k}| \approx \xi-1} B\left\langle\psi_{\mathbf{k}}^{*} \psi_{\mathbf{k}}\right\rangle / A \propto \xi^{-3} k_{B} T_{c} \chi_{0} B / A \propto \varepsilon^{3 \nu-\gamma-2 \beta}
$$

Since the equilibrium value of the order parameter below $T_{c}$ is given by $\left|\psi_{0}\right|^{2}=$ $-A / 2 B$, we can express (31) in terms of the Ginzburg factor $\Delta_{G}$ :

$$
1 \gg \Delta_{G}=\frac{1}{4 \pi e} k_{B} T_{c} \chi_{0} \xi^{-3}\left|\psi_{0}\right|^{-2} \propto \varepsilon^{3 v-(2-\alpha)}
$$

which is the well known Ginzburg criterion ${ }^{2}$ for the critical range. From this point of view, the Ginzburg criterion (32) indicates that the harmonic fluctuation terms $\left\langle\psi_{k}^{*} \psi_{k^{\prime}}\right\rangle$ are large compared with the anharmonic corrections. The generalized Landau theory (1) is applicable to systems that do not obey the scaling relation $\gamma+2 \beta=3 v$. From the Josephson inequality ${ }^{44} 3 v \geq 2-\alpha$ and the scaling relation $\gamma+2 \beta=2-\alpha$ which is a direct consequence of (1), we see that $\Delta_{G}$ is finite in the immediate vicinity of $T_{c}$. Analogous arguments can be presented below $T_{c}$ and also lead to the Ginzburg criterion. Thus according to (32), long-wavelength fluctuations $(|\mathbf{k}| \approx 1 / \xi)$ are statistically independent in the critical range.

The above considerations were based on (18), which is derived from (6) and (7) 
with anharmonic terms in (1) neglected, and the assumption that only wavevectors $\sim \xi^{-1}$ should be taken into the $\mathbf{k}$ sum (31). Revzen $^{45}$ has recently questioned the validity of (6) and (7), and based on a gauge-invariant formulation has proposed a new zeroth-order form, i.e., ignoring anharmonic terms in (1):

$$
\left\langle\psi_{\mathbf{k}}^{*} \psi_{\mathbf{k}}\right\rangle=\left[\exp \left(2 k_{B} T_{c} \chi_{k}\right)^{-1}-1\right]^{-1}
$$

which reduces to (18) for $2 k_{B} T_{c} \chi_{k} \gg 1$, and eliminates any short-wavelength divergences in the $\mathbf{k}$ integrals. The effect of (33) in an integral over $\mathbf{k}$ is to introduce an effective cutoff, $\chi_{\mathrm{k}}^{-1}=2 k_{B} T_{c}$, which is independent of the temperature difference and corresponds to the Brout criterion ${ }^{46}$ for the critical range. Integrating (31) to a temperature-independent cutoff, we get $\Delta_{B} \propto \varepsilon^{-2 \beta}$ which diverges at $T_{c}$. Thus according to the Brout criterion, our factorization (16) would not be allowed in the immediate vicinity of $T_{c}$. However, in the classical Landau theory Hohenberg ${ }^{46}$ has argued that the Ginzburg criterion, and not the Brout criterion, is the proper estimate of the classical range ; and the Ginzburg criterion has been used by Ferrell ${ }^{47}$ to estimate the anharmonic terms in the classical range. We have seen in the calculation for the thermal conductivity, and later for the sound absorption, that only $|\mathbf{k}| \sim 1 / \xi$ are important in the integrals over $\mathbf{k}$. Therefore we assume that in helium the Ginzburg criterion and not the Brout criterion is the correct one to estimate the factorization effects (31). With this proviso, we shall continue to ignore the anharmonic corrections.

\section{FIRST-SOUND ABSORPTION IN HELIUM}

The first calculation of the first-sound absorption near the superfluid transition in helium was performed by Landau and Khalatnikov (LK) ${ }^{14}$ using the classical time-dependent Landau equation (3). The general idea of LK was that the relaxation of the order parameter provided the dominant mechanism for sound absorption below $T_{c}$, but LK did not recognize that because of fluctuations a similar mechanism is applicable above $T_{c}$. Levanyuk ${ }^{15}$ remedied this oversight of LK and considered sound absorption brought about by the interaction of the sound wave with time-dependent fluctuations in the order parameter below and above $T_{c}$ in the classical range. For helium there is no classical range, and we consider now the calculation of the first-sound absorption in the critical range.

\subsection{Above the Superfluid Transition}

The sound absorption can be expressed ${ }^{36,48}$ in terms of the commutator of the perturbed Hamiltonian, which is in turn related by the fluctuation-dissipation theorem to an anticommutator correlation function. The resultant anticommutator involves a four $-\psi$ correlation function, which can be factorized and evaluated as in Section 3. In this manner, we can calculate the contribution to the sound absorption from order-parameter fluctuations if the coupling Hamiltonian between the sound waves and the order-parameter fluctuations is known. 
Consider a small change in sound-wave pressure $\delta p$; we assume that the perturbed energy at constant $\psi$ and $S$ is given by

$$
\mathscr{H}_{1}=\left(\frac{\delta F}{\delta p}\right)_{\psi, S} \delta p=\int d \mathbf{r}\left[\left(\frac{\partial A}{\partial p}\right)_{s}|\psi|^{2}+\left(\frac{\partial A_{0}}{\partial p}\right)_{s}|\nabla \psi|^{2}\right] \delta p
$$

where $F$ is the free energy (1) with anharmonic terms neglected. Ignoring the gradient term, we write the coupling Hamiltonian density as

$$
H_{1}(x)=G|\psi(x)|^{2} \delta p(x)
$$

where $x=(r, t)$ and the temperature-dependent vertex $G$ is

$$
G(\varepsilon)=\left(\frac{\partial\left(2 \chi_{0}^{-1}\right)}{\partial T_{c}}\right)_{s} \frac{\partial T_{c}}{\partial p}=\gamma \frac{\partial T_{c}}{\partial p}\left(2 \varepsilon T_{c} \chi_{0}\right)^{-1} C_{\chi} / C_{p} \propto \varepsilon^{\alpha+\gamma-1}
$$

If $A$ depends only on $T-T_{c}(p)$ and not on $p$ explicitly, the specific heat $C_{\chi}$ at constant susceptibility, $\chi_{0}=(2 A+\cdots)^{-1}$, is given by the derivative of the entropy parallel to the $\lambda$-line in the $p-T$ plane. Then in helium, ${ }^{33}$ we find $C_{\chi}=5$ joule/ $\mathrm{gm}^{\circ} \mathrm{K}$.

If the pressure variation is taken to be $\delta p(x)=p e^{i k x}$, then the amplitude attenuation is given by $\alpha=\dot{E} / 2 c E=\rho c \dot{E} / p^{2}$, where $E=\frac{1}{2} \rho v_{0}^{2}$ is the total energy density of the sound wave, $v_{0}=p / \rho c$ is the amplitude of the fluid velocity, $\rho$ is the mass density, $c$ is the sound speed, and $\dot{E}$ is the energy dissipation per unit volume. By the time-dependent perturbation theory and the fluctuation-dissipation theorem, ${ }^{36,48}$ we can write the first-sound amplitude attenuation as

$$
\begin{gathered}
\alpha=\frac{\rho c}{p^{2}} \cdot \frac{\omega}{4 h} \sum_{x} e^{i k x}\left\langle\left[H_{1}(x), H_{1}(0)\right]\right\rangle \\
=\frac{\rho c}{p^{2}} \cdot \frac{\omega^{2}}{4 k_{B} T_{c}} \sum_{x} e^{i k x}\left\langle H_{1}(x) H_{1}(0)\right\rangle \\
\sum_{x} \Rightarrow \int d \mathbf{r} \int d t
\end{gathered}
$$

Substituting (35) into (37) and factorizing the resulting four- $\psi$ correlation function as in (16), we obtain

$$
\frac{\alpha}{\omega^{2}}=\frac{\rho c G^{2}}{4 k_{B} T_{c}} \sum_{k^{\prime}}\left\langle\psi_{k^{\prime}}^{*} \psi_{k^{\prime}}\right\rangle\left\langle\psi_{k^{\prime}+k}^{*} \psi_{k^{\prime}+k}\right\rangle
$$

which like (17) can be represented by Fig. 1. Substituting (20) in (38), performing the $\omega^{\prime}$ integration, and taking the limit $\omega \tau_{0} \ll 1,|\mathbf{k}| \xi \ll 1, \omega=c|\mathbf{k}|$, we find that

$$
\frac{\alpha}{\omega^{2}}=\frac{\rho c G^{2}}{2 \pi^{2}} k_{B} T_{c} \int d \mathbf{k}^{\prime}\left|\mathbf{k}^{\prime}\right|^{2} \chi_{\mathbf{k}^{\prime}}^{2} \Gamma_{\mathbf{k}^{\prime}}^{-1}
$$

Using (36) we find the final form for the amplitude attenuation:

$$
\frac{\alpha}{\omega^{2}}=\frac{\gamma^{2}}{128 \pi}\left(\frac{1}{T_{c}} \frac{\partial T_{c}}{\partial p}\right)^{2} \rho c k_{B} T_{c}\left(\frac{C_{\chi}}{C_{p}}\right)^{2} \frac{\tau_{0}}{\xi^{3} \varepsilon^{2}} \propto \varepsilon^{3 v-2+2 \alpha} \tau_{0}
$$


At $T_{1}=T_{c}+10^{-5}{ }^{\circ} \mathrm{K}$, with $\tau_{0}$ given by $(25),\left(C_{x} / C_{p}\right)=0.35,\left(\partial T_{c} / \partial p\right)^{-1}=$ $114 \mathrm{~atm} /{ }^{\circ} \mathrm{K},{ }^{49} \xi=(1.6 \AA) \varepsilon^{-\frac{3}{3}},{ }^{40}$ and $\gamma=\frac{4}{3}$, we obtain from (40)

$$
\alpha / \omega^{2}=2 \times 10^{-14} \mathrm{sec}^{2} / \mathrm{cm} \quad \text { at } \quad T_{1}=T_{c}+10^{-5}{ }^{\circ} \mathrm{K}
$$

This value (41) is smaller by a factor of 8 than the experimental results of Barmatz and Rudnick. ${ }^{50}$ The reason for this discrepancy is not clear, although there are several doubtful numerical factors (e.g., $\gamma \neq \frac{4}{3}$ ) which may affect (41) by a factor of 3. The predicted temperature dependence $\alpha(\varepsilon) / \omega^{2}=1 \times 10^{-18} \varepsilon^{-1} \mathrm{sec}^{2} / \mathrm{cm}^{-1}$, does not agree with the $\varepsilon^{-\frac{1}{2}}$ dependence measured ${ }^{50}$ over one decade above $T_{1}=T_{c}+10^{-4}{ }^{\circ} \mathrm{K}$. However, at $22 \mathrm{kHz}$ and $T_{1}$ we find $\omega \tau_{0}=1.9$, which is beyond the range of validity of (40). In general for $\omega \tau_{0} \approx 1$, the absorption peak can be expected to be rounded, ${ }^{14}$ and this has a great influence on the apparent critical exponent of $\alpha$, but relatively little influence on the magnitude of $\alpha$. One can check this possible explanation of the $22 \mathrm{kHz}$ data by measuring $\alpha$ in the same temperature range with a lower frequency.

There have been other attempts to explain the first-sound absorption above $T_{c}$. In the theory of Wong, ${ }^{19}$ the inherent asymmetry of the Marcelja ${ }^{51}$ model for a superconducting transition is exploited to give $\alpha / \omega^{2} \propto \varepsilon^{-\frac{1}{2}}$ above $T_{c}$ and $\alpha / \omega^{2} \propto \varepsilon^{-1}$ below $T_{c}$. Below $T_{c}$, the theory of Wong is similar to $\mathrm{LK} ;{ }^{14}$ but above $T_{c}$, part of the anharmonic corrections neglected in the present calculation is included in a self-consistent Hartree approximation. ${ }^{51}$ A difficulty of this approach is the reconciliation of the predicted $\tau_{0}(\varepsilon) \propto \varepsilon^{-\frac{1}{2}}$ and the $\tau_{0}(\varepsilon) \propto \varepsilon^{-1}$ dependence obtained from the thermal conductivity measurements. Above $T_{c}$, Tsuzuki ${ }^{52}$ obtained $\alpha / \omega^{2} \propto \varepsilon^{-\frac{1}{5}}$ by considering the effect of the decay of a first-sound mode into two first-sound modes via a vertex of three order-parameter fluctuations. A difficulty of Tsuzuki's approach is the neglect of the decay of a first-sound mode into two-order parameter fluctuations (Fig. 1), which is the dominant contribution to the anomalous thermal conductivity. Szepfalusy ${ }^{53}$ and Ichiyanagi ${ }^{54}$ have suggested the form $\alpha /|\mathbf{k}|^{2} \propto \varepsilon^{-1} f(|\mathbf{k}| \xi)$ and fitted $\alpha \propto|\mathbf{k}|^{\frac{1}{3}} \varepsilon^{-\frac{1}{2}}$ above $T_{c}$. From the present approach, one may get such an additional factor $(|\mathbf{k}| \xi)^{-\frac{3}{4}}$ if the $\mathbf{k}^{\prime}$ integration in (39) diverges for low $\left|\mathbf{k}^{\prime}\right|$. But such is not the case for our approximations, which give $f=1$ above $T_{c}$.

\subsection{Below the Superfluid Transition}

Since the order parameter has a nonzero mean value $\psi_{0}$ below $T_{c}$, it is convenient in helium to take $\psi_{0}$ "lined up" along the real axis, $\operatorname{Im} \psi_{0}=0$, and to separate the fluctuations about $\psi_{0}$ into amplitude (longitudinal, $\|$ ) and phase (transverse, $\perp$ ) fluctuations. The frequency spectrums are like (20), apart from a factor of 2 :

where

$$
\begin{gathered}
\left\langle\left(\psi_{k}^{\|, \perp}\right)^{2}\right\rangle=k_{B} T_{c} \chi_{k}^{\|, \perp} \\
\chi_{k}^{\|, \perp}=\chi_{k}^{\|, \perp} \cdot 2 \Gamma_{k}^{\|, \perp} /\left[\omega^{2}+\left(\Gamma_{k}^{\|, \perp}\right)^{2}\right]
\end{gathered}
$$

$$
\left\{\begin{array}{l}
\Gamma_{k}^{\|}=\left(R / 2 \chi_{k}\right)=\Gamma_{0}(-\varepsilon)\left(1+|\mathbf{k}|^{2} \xi^{2}\right) \\
\Gamma_{\mathbf{k}}^{\perp}=\left(R / 2 \chi_{\mathbf{k}}^{1}\right)=\Gamma_{0}(-\varepsilon)|\mathbf{k}|^{2} \xi^{2}
\end{array}\right.
$$


with $\Gamma_{0}(-\varepsilon)=1 / \tau_{0}(-\varepsilon)=\left[R / 2 \chi_{0}(-\varepsilon)\right]=(-2 R A+\cdots)$ and $\xi=\left(2 A_{0} \chi_{0}\right)^{\frac{1}{2}}$. We ignore the damping due to the phase fluctuations as it vanishes in the longwavelength limit. Henceforth all fluctuations are amplitude fluctuations and the superscript $\|$ will be suppressed.

In (38), we separate out the contribution of $\psi_{0}$ and write

$$
\frac{\alpha}{\omega^{2}}=\frac{\rho c G^{2}}{4 k_{B} T_{c}}\left[\psi_{0}^{2}\left\langle\psi_{k}^{2}\right\rangle+\left\langle\psi_{k}^{2}\right\rangle \psi_{0}^{2}+\sum_{k^{\prime}}^{\prime}\left\langle\psi_{k^{\prime}}^{2}\right\rangle\left\langle\psi_{k^{\prime}+k}^{2}\right\rangle\right]
$$

where the sum includes only the fluctuations. The first two terms correspond to the relaxation of the equilibrium order parameter, which was considered by LK in the classical range, and yields the amplitude attenuation [substitute (42) into (43)]

$$
\begin{aligned}
\alpha_{0} / \omega^{2} & =\rho c G^{2} \psi_{0}^{2} \chi_{0} \tau_{0}\left(1+\omega^{2} \tau_{0}^{2}\right)^{-1}\left(C_{\chi} / C_{p}\right)^{2} \\
& \approx \frac{3}{2} \gamma^{2} \rho c\left(\frac{1}{T_{c}} \frac{\partial T_{c}}{\partial p}\right)^{2} T_{c} \Delta C_{p}\left(\frac{C_{\chi}}{C_{p}}\right)^{2} \tau_{0}(-\varepsilon) \propto \varepsilon^{\alpha} \tau_{0} \quad\left(\omega \tau_{0} \ll 1\right)
\end{aligned}
$$

where the last equation follows from Eq. (4) of Stauffer, ${ }^{11} \Delta C_{p}$ is the difference in the specific heat across $T_{c}$ and $\tau_{0}(-\varepsilon)$ is the relaxation time below $T_{c}$. If we use $\tau_{0}(-\varepsilon) \propto \varepsilon^{-1}$, as above $T_{c}$, we see that $\alpha_{0} / \omega^{2} \propto \varepsilon^{-1}$. The amplitude attenuation due to fluctuations, $\alpha_{1} / \omega^{2}$, which is given by the third term in (43), is present on both sides of $T_{c}$, and has the same temperature dependence as (40). However below $T_{c}$, the correlation function $\chi_{k}$ contains not only a central relaxation peak [Eq. (42)] but also two second-sound peaks ${ }^{37}$ centered around $\pm \omega_{2}= \pm c_{2}|\mathbf{k}|$. In other words, Eq. (4) is probably incomplete below $T_{c}$. Therefore we must modify (42b), and as an example we write

$$
\frac{\chi_{k}}{\chi_{\mathbf{k}}}=z_{c} \frac{2 \Gamma_{\mathbf{k}}}{\omega^{2}+\Gamma_{\mathbf{k}}^{2}}+z_{s} \frac{\gamma_{\mathbf{k}}}{\left(\omega-\omega_{2}\right)^{2}+\gamma_{\mathbf{k}}^{2}}+z_{s} \frac{\gamma_{\mathbf{k}}}{\left(\omega+\omega_{2}\right)^{2}+\gamma_{\mathbf{k}}^{2}}
$$

where $2 \gamma_{k}=D_{2}|\mathbf{k}|^{2}$ gives the damping of second sound and $z_{c}+z_{s}=1$ according to the sum rule (21). If we use (45) instead of (42b) in deriving (44), we see that (44) must be multiplied by the factor $z_{c} \leq 1$. If the second-sound peaks are small, i.e., $z_{s} \ll z_{c} \approx 1$, then $\alpha_{1} / \alpha_{0} \approx \Delta_{G}(e / 32)<1$, and $\alpha_{0}$ according to (44) and (25) turns out to be 5 times larger than the experimental data. ${ }^{50}$ In other words, (25) gives a $\tau_{0}(-\varepsilon)$ about 5 times larger than the estimate of Barmatz and Rudnick. ${ }^{50}$ In the limit $z_{c} \ll z_{s} \approx 1$, the amplitude attenuation $\alpha \approx \alpha_{1}$ is mainly due to the secondsound peaks, which is the main mechanism considered in the mode-mode coupling theory $;^{38}$ and $\alpha_{1}$ can be obtained from (39) if we replace $\Gamma_{\mathbf{k}}$ by $\gamma_{k}=\frac{1}{2} D_{2}|\mathbf{k}|^{2}$. In this manner, we get

$$
\frac{\alpha(-\varepsilon)}{\omega^{2}} \approx \frac{\alpha_{1}(-\varepsilon)}{\omega^{2}}=\frac{\gamma^{2}}{128 \pi} p c k_{B} T_{c}\left(\frac{1}{T_{c}} \frac{\partial T_{c}}{\partial p}\right)^{2}\left[D_{2}(-\varepsilon) \xi(-\varepsilon) \varepsilon^{2}\right]^{-1}\left(C_{\chi} / C_{p}\right)^{2}
$$

If we fit $\alpha_{1}(-\varepsilon) \omega^{2}$ to the measured amplitude attenuation at $22 \mathrm{kHz}$, we can invert (46) and deduce $D_{2}(-\varepsilon)$. Using $\alpha(-\varepsilon) / \omega^{2} \approx 0.6 \times 10^{-18}|\varepsilon|^{-1} \mathrm{sec}^{2} / \mathrm{cm},{ }^{50}$ and $\xi(-\varepsilon)=1.2 \AA|\varepsilon|^{-\frac{2}{3}, 40}$ we obtain from (46) a $D_{2}$ smaller than the measured one ${ }^{55}$ by 
a factor of 50 . This result is outside our numerical inaccuracies. We conclude that the first-sound damping in $\mathrm{He} \mathrm{II}$ is mainly due to the $\mathrm{LK}$ relaxation of the equilibrium order parameter [Eq. (44) multiplied by an unknown $z_{c} \approx 1$ ] and not due to the decay of a first-sound mode into two order-parameter fluctuations with a small central peak and large second-sound modes.

From dynamic scaling, ${ }^{20}$ we see that $\tau_{0}(-\varepsilon) \propto \zeta(-\varepsilon) / c_{2}(-\varepsilon) \propto|\varepsilon|^{-1}\left(C_{p}^{-}\right)^{\frac{1}{2}}$. Assuming $\alpha_{0}(-\varepsilon) \propto \alpha_{1}(-\varepsilon)$, we find $\alpha(-\varepsilon) \propto|\varepsilon|^{-1}\left(C_{p}^{-}\right)^{-\frac{3}{2}}$ and $D_{2}(-\varepsilon) \propto$ $|\varepsilon|^{-t}\left(C_{p}^{-}\right)^{-\frac{1}{2}}$. Ferrell et al. ${ }^{12}$ obtained the same expression for $D_{2}(-\varepsilon)$, but for $\alpha$ they get $\alpha(-\varepsilon) \propto|\varepsilon|^{-1}\left(C_{p}^{-}\right)^{-\frac{1}{2}}$, which disagrees with our result. The discrepancy is traced back to the assumption by Ferrell et al. ${ }^{12}$ that the second viscosity $\zeta_{2}$ is the dominant contribution to the damping of both first and second sound, which leads to $\alpha \propto D_{2} \xi$. From (46) we see that the first-sound absorption is inversely proportional to the damping of the critical mode, which is consistent with other transition (see next section). Note that our logarithmic dependence for $\alpha$ agrees with that of Ichiyanagi ${ }^{54}$ if we assume isothermal sound propagation.

We can apply the present method directly to the calculation of the secondsound amplitude attenuation $\alpha_{2}$ and obtain $\alpha_{2}=\dot{E}_{2} / c_{2} \rho_{s} v_{s}^{2}=\left(c_{2} / \rho_{s} S^{2}\right)\left[\dot{E}_{2} /(\delta T)^{2}\right]$, where $\dot{E}_{2}$ is the second-sound energy dissipation per unit volume, and $\delta T=$ $c_{2} v_{s} / S$ is the temperature variation. If we only relate $\alpha_{2}$ to $\alpha$, then we expect that the problems connected with $\chi_{k}$ would cancel out and that the second-sound damping $D_{2}=2 c_{2}^{3} \alpha_{2} / \omega^{2}$ can be expressed directly in terms of the measured $\alpha$. In calculating the energy dissipation $\dot{E}_{2}$, we replace $H_{1}(x)=(\partial f / \partial p)_{\psi, s} \cdot \delta p$ by $H_{2}(x)=$ $(\partial f / \partial T)_{\psi, p} \cdot \delta T$; and the respective vertex functions are related by $G / G_{2}=$ $\left(\partial T_{c} / \partial p\right)\left(C_{\chi} / C_{p}^{-}\right)$. In this manner we obtain for the ratio of the amplitude attenuations

$$
\frac{\alpha_{2}}{\alpha}=\frac{c_{2} / \rho_{s} S^{2}}{\rho c}\left(\frac{G_{2}}{G}\right)^{2}=\frac{c_{2} / \rho_{s} S^{2}}{\rho c\left(\partial T_{c} / \partial p\right)^{2}}\left(\frac{C_{p}^{-}}{C_{\chi}}\right)^{2}
$$

Using $c_{2}^{2}=\left(Y_{c} S^{2} \rho_{s} / \rho C_{p}^{-}\right)$, we can write

$$
\begin{aligned}
D_{2}(-\varepsilon) & =\frac{2}{c}\left(\frac{T_{c} S}{\rho C_{\chi}}\right)^{2}\left(\frac{\partial T_{c}}{\partial p}\right)^{-2} \cdot \frac{\rho_{s}(-\varepsilon)}{\rho} \cdot \frac{\alpha(-\varepsilon)}{\omega^{2}} \\
& =0.4 \times 10^{-4}|\varepsilon|^{-\frac{1}{3}} \mathrm{~cm}^{2} / \mathrm{sec}
\end{aligned}
$$

where we have used $\rho_{s}(-\varepsilon) / \rho=2.40|\varepsilon|^{\frac{2}{3}}$ and $\alpha(-\varepsilon) / \omega^{2}=0.6 \times 10^{-18}|\varepsilon|^{-1} \sec ^{2} /$ $\mathrm{cm},{ }^{50}$ to obtain (47b). This result $(47 \mathrm{~b})$ is by about a factor of 2 smaller than the measured ${ }^{55} D_{2}(-\varepsilon)=1.02 \times 10^{-4}|\varepsilon|^{-\frac{1}{5}} \mathrm{~cm}^{2} / \mathrm{sec}$.

Usui $^{56}$ has recently generalized the classical Pitaevskii ${ }^{57}$ theory of superfluidity to the critical range. Upon fitting the calculated first-sound damping to experiments, he finds in his theory a similar expression for $D_{2}(-\varepsilon)$ as in (47b) but with the coefficient 0.7 in place of 0.4 . The generalized Pitaevskii theory is more complicated than the present approach, e.g., in Reference 56 the equation of motion for the order parameter reduces to the generalized time-dependent Landau equation (4) if only the resistive part of $B$ is retained ; and in principle the generalized Pitaevskii theory takes into account density and entropy fluctuations, which have 
been ignored in the present theory. Nevertheless, because of its simplicity, the present theory can display the physical processes involved better than the Pitaevskii approach. Furthermore, we have shown that the present theory is easily applied to helium and other systems (see next section) above $T_{c}$, which is yet to be done in the generalized Pitaevskii theory.

\section{SOUND ABSORPTION ABOVE THE FERROMAGNETIC TRANSITION}

If we apply our sound-absorption formalism to other second-order phase transitions below $T_{c}$, we have the same problems as in helium. For example, in ferromagnets there are spin-wa ve peaks in $\chi_{k}$, and in iron ${ }^{58}$ and nickel ${ }^{59}$ the central peak is too small to be observable. Therefore we discuss only the paramagnetic case $T>T_{c}$.

The paramagnetic state obeys a more general equation than (4), i.e., a diffusive term must be added $:^{36}$

$$
\left(\frac{\partial}{\partial t}-D \nabla^{2}\right) \psi=-R \cdot L\{\psi\} \psi
$$

Beginning with (48) instead of (4) we see that (19) is replaced with

$$
\Gamma_{\mathbf{k}}=\tau_{0}^{-1}\left(1+|\mathbf{k}|^{2} \xi^{2}\right)+D|\mathbf{k}|^{2}
$$

where $\tau_{0}$ is as before the zero-wavevector relaxation time. In general, if we substitute (49) into (39) the net result is that $\tau_{0}$ in (40) must be replaced by $1 / \Gamma\left(|\mathbf{k}|=\xi^{-1}\right)$ apart from numerical factors. In the physically interesting limit $D \xi^{-2} \gg \tau_{0}^{-1}$, the relaxation term can be ignored and we have, as in (46)

$$
\frac{\alpha}{\omega^{2}}=\frac{s^{2} \gamma^{2}}{128 \pi} \rho c k_{B} T_{c}\left(\frac{1}{T_{c}} \frac{\partial T_{c}}{\partial p}\right)^{2}\left[D(\varepsilon) \xi(\varepsilon) \varepsilon^{2}\right]^{-1}\left(\frac{C_{x}}{C_{p}}\right)^{2}
$$

where we have written (50) for a general-order parameter of rank $s$ ( $s=1$ for scalar, $s=2$ for spinor, $s=3$ for 3-dimensional vector, etc.) which obeys (48). From dynamic scaling, ${ }^{13}$ we can take for the paramagnetic state $D(\varepsilon) \propto \xi^{-\frac{1}{2}+(\eta / 2)}$, where $\eta$ is the critical exponent measuring the deviation of the spin-spin correlation function from the classical Ornstein-Zernike form. Thus we find

$$
\alpha(\varepsilon) / \omega^{2} \propto \varepsilon^{-2+\frac{1}{2}(1+\eta) v+2 \alpha}
$$

If we take $v \approx \frac{2}{3}$ and $\eta \approx 0$, then we get $\alpha(\varepsilon) \propto \varepsilon^{-\frac{5}{3}}$ in agreement with Kawasaki. ${ }^{60}$ Equations (40), (51), and (50) are very similar to the result from the mode-mode calculation of Laramore and Kadanoff, ${ }^{61}$ apart from the numerical factor $s^{2} / 128 \pi$. The numerical discrepancy arises in Reference 61 from the estimate of the $q$ integral $\sum_{\mathrm{q}}$ by $\xi^{-3}$.

Quantitatively, we evaluate (50) at $\varepsilon=10^{-2}$ with the values for nickel $D=0.53 \times 10^{14} \AA^{2} / \mathrm{sec}^{59} \xi=36 \AA,{ }^{59}\left(\partial T_{c} / \partial p\right)=0.37^{\circ} \mathrm{K} / \mathrm{kbar}^{62} \gamma=\frac{4}{3}, c=5$ $\mathrm{km} / \mathrm{sec}, C_{x} / C_{p} \approx 1$ because of the large background, and obtain

$$
\alpha\left(\varepsilon=10^{-2}\right) / \omega^{2}=s^{2} \cdot 3 \times 10^{-21} \mathrm{sec}^{2} / \mathrm{cm} .
$$


Golding and Barmatz ${ }^{63}$ measured in nickel $\alpha=0.1 \mathrm{~dB} / \mathrm{cm}$ at $\varepsilon=10^{-3}$ and $\omega=60 \mathrm{MHz}$. Extrapolating their data with their critical exponent of 1.4 for $\alpha$, we obtain

$$
\alpha\left(\varepsilon=10^{-2}\right) / \omega^{2}=3.3 \times 10^{-21} \mathrm{sec}^{2} / \mathrm{cm} .
$$

Equation (52) agrees very well with (53) if there exists only one degree of freedom $(s=1)$ as in an Ising model. However, because of the various extrapolations and numerical uncertainties, an agreement between (52) and (53) better than a factor of 4 is fortuitous. Nevertheless, the agreement of (52) and (53) in order of magnitude is sufficient to confirm our rather surprising factor of $s^{2} / 128 \pi$ in (50), which is absent in the calculations using mode-mode coupling. ${ }^{61}$ As was noted in Reference 63 , if the values of the critical exponents as measured in nickel, $\eta=0.08,{ }^{59}$ $\alpha=0.10,{ }^{64} v=(2-\alpha) / 3 \approx 0.64$, were inserted into $(51)$, we obtained

$$
\alpha(\varepsilon) / \omega^{2} \propto \varepsilon^{-1.47}
$$

which is in good agreement with the experimental value ${ }^{63} \varepsilon^{-1.4 \pm 0.1}$. However, we note that the measured critical exponent ${ }^{59}$ for the diffusion constant $0.5 \pm 0.05 \neq$ $\frac{1}{2} v(1+\eta) \approx 0.35$, which casts some doubt on the reliability of (54).

Our Ansatz [(34)-(36)] for the coupling energy obviously cannot be correct in systems where an applied pressure above $T_{c}$ produces a nonzero equilibrium order-parameter, e.g., the liquid-gas transition; the only coupling that we have taken into account is a shift in the transition temperature. Also for magnetic systems, our results and those of Reference 61 disagree with some experiments, e.g., in $\mathrm{RbMnF}_{3}$. For discussion in greater detail, see Reference 61.

\section{CONCLUDING REMARKS}

The present scarcity of experimental data precludes any conclusive confirmation of our results. If future research should agree with our results, then the present approach seems to us to be a useful complement of the existing calculations of critical dynamic properties. Unlike the dynamic-scaling hypothesis ${ }^{12,13}$ which relates the critical exponents of $\tau_{0}$ or $D$ to the static exponents and the dispersion relation for the critical excitations below $T_{c}$, the present approach cannot predict the divergences of $\tau_{0}$ and $\xi$, which must be taken from experiments. On the other hand, the phenomenological approach can be checked quantitatively, i.e., as to the magnitude (critical coefficient) as well as the temperature dependence (critical exponent) of the critical behavior. The order-of-magnitude agreement between our results and available experiments confirms our numerical factors (e.g., $s^{2} / 128 \pi$ ) which are absent in the results in Reference 61 from mode-mode coupling. Compared with mode-mode coupling ${ }^{38,61}$ the present approach is simpler and quantitatively more accurate; compared with the dynamic-scaling approach, ${ }^{12,13}$ it is more instructive in giving detailed information as to which processes and which wavevectors are important in a particular dynamic property.

The generalized Landau theory of second-order phase transition is thus shown to be useful in the critical range not only in calculating static properties but also dynamic properties via the time-dependent version. Further investigation is needed 
to understand the role of the anharmonic terms in the order-parameter fluctuations (particularly the cutoff problem) and the effect of the neglected fluctuations in energy and density.

\section{NOTES ADDED IN PROOF}

The high-frequency sound absorption in He I has been recently calculated by K. Kawasaki [Phys. Letters 31A, 165 (1970)] using the mode-mode coupling theory. For low frequencies, the result of Kawasaki agrees with our Eq. (38). At high frequencies, the main contribution to the absorption comes from shortwavelength $(\ll \xi)$ fluctuations which we have ignored. Hence our argument for the factorization of the $4 \psi$-correlation function (Fig. 1) is no longer valid for high frequencies.

Problems with logarithmic factors in a mode-mode coupling theory have been raised by $P$. Szepfalusy (private communication and to be published). Our result for the sound absorption is likewise unreliable as far as logarithmic factors are concerned. If the real part corresponding to the diagram of Fig. 1 is assumed to give the change $\Delta c$ in the sound speed [cf. H. S. Bennett, Phys. Rev. 185, 801 (1969)], then we find $\Delta c \propto C_{p}^{-2}$ in He I, whereas experimentally $\Delta c \propto C_{p}^{-1}$. Thus the sound absorption $\alpha$ is too small by a logarithmic factor. See discussion on page 613 .

\section{ACKNOWLEDGMENT}

We thank Dr. J. Jäckle, Prof. H. Schmidt, and Prof. H. Gould for helpful ideas and discussions.

\section{REFERENCES}

1. See for example L. D. Landau and E. M. Lifshitz, Statistical Physics (Pergamon Press, London, 1958), Chap. 14.

2. L. P. Kadanoff et al., Rev. Mod. Phys. 39, 395 (1967), and references therein.

3. M. E. Fisher, Rept. Progr. Phys. 30, 615 (1967).

4. P. Heller, Rept. Progr. Phys. 30, 731 (1967).

5. B. Widom, J. Chem. Phys, 43, 3892 (1965); 43, 3898 (1965).

6. L. P. Kadanoff, Physics 2, 263 (1966).

7. See for example P. G. DeGennes, Superconductivity of Metals and Alloys (W. A. Benjamin, New York, 1966).

8. D. J. Amit and M. Luban, Phys. Letters 27A, 487 (1968).

9. V. K. Wong, Phys. Letters 27A, 358 (1968).

10. S. Fisk and B. Widom, J. Chem. Phys, 50, 3219 (1969); J. S. Huang and W. W. Webb, J. Chem. Phys. 50, 3677 (1969); J. T. Ho and J. D. Litster, Phys. Rev. Letters 22, 603 (1969).

11. D. Stauffer, Z. Physik 221, 122 (1969).

12. R. A. Ferrell et al., Ann. Phys. (New York) 47, 565 (1968).

13. B. I. Halperin and P. C. Hohenberg, Phys. Rev. 177, 952 (1969).

14. L. D. Landau and I. M. Khalatnikov, Dokl. Akad. Nauk SSSR 96, 469 (1954); English transl. in Collected Papers of L. D. Landau, D. ter Haar, ed. (Pergamon Press, Oxford, 1965), p. 626.

15. A. P. Levanyuk, Zh. Eksperim. i Teor. Fiz. 49, 1304 (1965); English transl. Soviet Phys.-JETP 22, 901 (1966).

16. K. Kawasaki (to be published, private communication).

17. M. Suzuki, H. Ikari, and R. Kubo, J. Phys. Soc. Japan 26 (Suppl.), 153 (1969).

18. V. L." Pokrovskii and I. M. Khalatnikov, JETP Letters 9, 149 (1969); I. M. Khalatnikov, Zh. Eksperim. i Teor. Fiz. 57, 489 (1969). 
19. V. K. Wong, Phys. Letters 29A, 441 (1969).

20. C. DiCastro, F. Ferro-Luzzi, and J. A. Tyson, Phys. Letters 29A, 458 (1969).

21. B. D. Josephson, Phys. Letters 21, 608 (1969).

22. L. G. Aslamazov and A. I. Larkin, Fiz. Tverd. Tela 10, 1104 (1968); English transl. Soviet Phys.Solid State 10, 875 (1968). See also L. G. Aslamazov and A. I. Larkin, Phys. Letters 26A, 238 (1968); K. Yamaji, Phys. Letters 29A, 123 (1969); K. D. Usadel, Phys. Letters 29A, 501 (1969).

23. E. Abrahams and J. W. F. Woo, Phys. Letters 27A, 117 (1968).

24. A. Schmid, Z. Phys. 215, 210 (1968); Phys, Rev. 180, 527 (1969).

25. H. Schmidt, Z. Phys. 216, 336 (1968).

26. R. A. Ferrell, J. Low Temp. Phys. 1, 241 (1969).

27. R. Mountain and R. Zwanzig, J. Chem. Phys. 48, 1451 (1968).

28. G. Bergmann, Z. Phys. 225, 430 (1969).

29. H. J. Mikeska and H. Schmidt, Z. Physik 230, 234 (1970).

30. M. Archibald, J. M. Mochel, and L. Weaver, Phys. Rev. Letters 21, 1156 (1968).

31. G. Ahlers, Phys, Rev, Letters 21, 1159 (1968).

32. A. P. Levanyuk, Fiz. Tverd. Tela 5, 1776 (1963); English transl. Soviet Phys.-Solid State 5, 1294 (1964). See also J. A. Tyson, Phys. Letters 24A, 183 (1967); H. Stenschke and G. Falk, Z. Phys. 212, 308 (1968).

33. W. M. Fairbank and C. F. Kellers, in Critical Phenomena Proceedings of a Conference, Washington, D.C., 1965, M. S. Green and J. V. Senger, eds. (NBS Misc. Publ. No. 273), p. 71 ; M. J. Buckingham and W. M. Fairbank, in Progress in Low Temp. Physics, Vol. III, C. J. Gorter, ed. (North Holland, Amsterdam, 1961), p. 80.

34. G. Ahlers, Phys, Rev. Letters 23, 464 (1969).

35. V. K. Wong, Phys. Letters 28A, 248 (1968).

36. L. P. Kadanofi and P. C. Martin, Ann. Phys. (New York) 24, 419 (1963).

37. P. C. Hohenberg and P. C. Martin, Ann. Phys. (New York) 34, 291 (1965).

38. J. Swift and L. P. Kadanoff, Ann. Phys. (New York) 50, 312 (1968).

39. K. Kawasaki and T. Tsuzuki, Phys. Letters 28A, 30 (1968).

40. Yu. G. Mamaladze, Zh. Eksperim. i Teor. Fiz.52, 729 (1967); English transl., Soviet Phys.-JETP 25, 479 (1967). See also V. K. Wong, Phys. Letters 27A, 269 (1968).

41. H. Gould and V. K. Wong, Phys. Letters 31A, 364 (1970).

42. J. Wilkins, "Time-Dependent Fluctuation in the Order Parameter of $\mathrm{He}^{4}$ Above $T_{\lambda}$ " (preprint, Cornell University, Ithaca, N.Y.).

43. A. Eggington and A. Leggett (private communication).

44. B. D. Josephson, Proc. Phys. Soc. 92, 276 (1967).

45. M. Revzen, Phys. Letters 29A, 443 (1969).

46. P. C. Hohenberg in Conf. Fluctuations in Superconductors, Asilomar 1968, W. S. Goree and F Chilton, eds. (Stanford Research Institute, 1968), p. 305.

47. R. A. Ferrell, ibid., p. 239.

48. J. Jäckle, Z. Phys. 231, 262 (1970).

49. G. Ahlers, Phys. Rev. 170, 275 (1968).

50. M. Barmatz and I. Rudnick, Phys. Rev. 170, 224 (1968). For higher frequencies, see W. Heinicke, G. Winterling, and K. Dransfeld, Phys. Rev. Letters 22, 694 (1969).

5.1. S. Marcelja, Phys. Letters 28A, 180 (1968); S. Marcelja, W. E. Masker, and R. D. Parks, Phys. Rev. Letters 22, 124 (1969); Phys. Rev. 188, 745 (1969).

52. T. Tsuzuki, Progr. Theoret. Phys. 41, 1387 (1969); 42, 146E (1969).

53. P. Szepfalusy, Phys. Letters 27A, 128 (1968).

54. M. Ichiyanagi, Progr. Theoret. Phys. 42, 147 (1969).

55. J. A. Tyson, Phys. Rev. Letters 21, 1235 (1968).

56. T. Usui, Progr. Theoret. Phys. 41, 1603L (1969); T. Usui and J. Yamauchi (preprint, Nagoya University, Japan).

57. L. P. Pitaevskii, Zh. Eksperim. i Teor. Fiz. 35, 408 (1958); English transl., Soviet Phys._JETP 8, 282 (1959).

58. M. F. Collins, V. J. Minkiewicz, R. Nathans, L. Passell, and G. Shirane, Phys. Rev. 179, 417 (1969).

59. V. J. Minkiewicz, M. F. Collins, R. Nathans, and G. Shirane, Phys. Rev. 182, 624 (1969).

60. K. Kawasaki, Phys. Letters 26A, 543 (1968).

61. G. E. Laramore and L. P. Kadanoff, Phys. Rev. 187, 619 (1969).

62. T. Okamoto, H. Fujii, M. Tsurui, H. Fujiwara, and E. Tatsuneto, J. Phys. Soc. Japan 22, 337 (1967).

63. B. Golding and M. Barmatz, Phys. Rev. Letters 23, 223 (1969).

64. W. E. Maher and W. D. McCormick, Phys. Rev. 183, 573 (1969). 